\title{
Design and application of subaxial cervical pedicle screw placement guide device
}

\author{
QIANG WANG ${ }^{1}$, RUNLIN XING ${ }^{2}$ and YIWEN ZENG ${ }^{1}$ \\ ${ }^{1}$ Department of Orthopaedics, Nanjing First Hospital, Nanjing Medical University; ${ }^{2}$ Department of Orthopaedics, \\ Affiliated Hospital of Nanjing University of Chinese Medicine, Nanjing, Jiangsu 210000, P.R. China
}

Received August 27, 2017; Accepted May 11, 2018

DOI: $10.3892 /$ etm.2019.7479

\begin{abstract}
In the present study, a novel subaxial cervical pedicle screw placement guide device was designed and developed. In cervical specimens (C3-C7), a pedicle screw was inserted into the left pedicle using the guide device with a keyhole partial laminectomy and tapping technique, and the right pedicle by drilling using the Abumi technique. After removing the pedicle screws, the channel wall of each pedicle screw was probed with a pedicle probe. The vertebral body was then dissociated for direct observation of the screw channel. Among the 10 specimens, 2 of the 50 pedicles $(4 \%)$ in the guide device group were perforated. Screw placement failed in 8 of 50 pedicles (16\%) in the Abumi technique group. Significant differences were observed in the outcomes for the guide device and Abumi technique groups. The subaxial cervical pedicle screw placement guide device developed in the present study decreased the failure rate of pedicle screw placement.
\end{abstract}

\section{Introduction}

In cervical surgery, the pedicle screw technique has demonstrated reliable biomechanical compatibility and a high rate of bone fusion (1). However, accurate placement of the screw is difficult, as the outer diameter of the cervical pedicle is smaller than the thoracolumbar pedicle (2). Furthermore, the cervical pedicle has a large range of variation and adjoins the vertebral artery, nerve root and spinal cord (3) Pedicle perforation from any direction is associated with a considerable risk, intra-operative complications, including the rupture of the bone wall, may decrease the clamping force of the screw, and the rate of grade 2 and 3 screw perforation is $\sim 20 \%$ (4). A previous study has demonstrated a low rate of intra-operative complications associated with cervical pedicle screw fixation,

Correspondence to: Dr Yiwen Zeng, Department of Orthopaedics, Nanjing First Hospital, Nanjing Medical University, 68 Changle Road, Nanjing, Jiangsu 210000, P.R. China

E-mail: zengyiwen1961@163.com

Key words: subaxial cervical pedicle, pedicle screw, guide device but a high incidence of mild to moderate screw deviation, which may cause complications including the perforation of the pedicle wall, vertebral artery injury, nerve root injury and spinal cord injury (5).

Therefore, the present study performed a detailed applied anatomy investigation of the cervical pedicle and designed a novel subaxial cervical pedicle screw placement device to increase the accuracy of subaxial cervical pedicle screw placement and to reduce the occurrence of complications during screw placement.

\section{Materials and methods}

Structure of the guide device. The subaxial cervical pedicle screw placement guide device comprises two parts: The positioning and orientation. Its structural features are presented in Fig. 1. The upper part of a fixed positioning pin is connected to an arcuate sliding groove, the center of which is located $1.5 \mathrm{~mm}$ from the tip of the fixed positioning pin. The sliding groove is marked with a $0-45^{\circ}$ graduation, and two tightening screws are present at 30 and $45^{\circ}$, respectively. These two tightening screws are used to tighten the sliding groove and fix the working sleeve. Inside the sliding groove, there are four working sleeve slots at $30,35,40$ and $45^{\circ}$, in which to place the working sleeve. The guide device is made of surgical steel and produced by Suzhou Qingniu Medical Device Co., Ltd. (Suzhou, China) in strict accordance with the principles and structural features of invention patent no. ZL200810123915.6.

Preparation of specimens. Subaxial cervical specimens of 10 antiseptically treated adult male human cadavers were selected (aged 60-80 years). A longitudinal posteromedial incision was made to bilaterally expose the outer edge of the facet joints. In total, 100 cervical pedicles of 50 vertebral bodies (C3-C7) were measured. The inclination and sagittal angles of the pedicle were measured via the X-ray examination (Fig. 2). The 100 cervical pedicles were randomly divided into two groups according to the different screw-setting techniques; in the guide device group, the Subaxial Cervical Pedicle Screw Placement Guide Device was used, and in the control group, the Abumi technique was applied.

Subaxial cervical pedicle screw placement guide device. The subaxial cervical pedicle screw placement guide device (Fig. 3) 
was used in combination with a keyhole partial laminectomy and tapping technique (6). A keyhole fenestration of $\sim 5 \mathrm{~mm}$ was created between the fixed vertebral body and adjacent vertebral plate for placement of the locator. After identification of the medial, superior and inferior borders of the pedicle, the positioning pin was fixed in the center of the medial border of the pedicle. The direction of the working sleeve was identified by regulating the direction of the fixed positioning pin and the angle of the sliding groove. The inclination angle of the working sleeve was $40-45^{\circ}$ at $\mathrm{C} 3-\mathrm{C} 6$ and $30-40^{\circ}$ at $\mathrm{C} 7$. The sagittal angle of the working sleeve was parallel to the vertebral upper endplate (caudally inclined to $\mathrm{C} 3-\mathrm{C} 4$ at an angle of $\sim 10^{\circ}$, resembling a pointed cone; cephalically inclined to C6-C7 at an angle of $\sim 10^{\circ}$ and perpendicular to the posterolateral surface of the $\mathrm{C} 5$ vertebral body). After the working sleeve had been fixed with the tightening screw, the heading device of the pedicle was rotated in the pedicle. Finally, a pedicle screw of suitable diameter and length was implanted.

Abumi technique. After determining the position of the nail, high-speed grinding is used to drill the bone cortex of the nail point to create a suitable round hole. The diameter of the hole is approximately equal to the screw neck, so that the entrance of the pedicle canal may be seen directly. The small probe was moved into the vertebral body by the pedicle of the medullary cavity under X-ray $\mathrm{C}$ arm monitoring, after the pedicle screw entered slowly into the pedicle. An inward tilt angle should be applied combined with pre-operative computed tomography (CT) and intra-operative assessment. Intra-operative lateral $\mathrm{X}$-ray monitoring was used to ensure that the probe is located in the pedicle and along the probe, and inserted into the location and direction of tapping. Combined with pre-operative $\mathrm{CT}$, the distance of the pedicle to the vertebral anterior, the pedicle medullary cavity diameter and intraoperative examination were used select the appropriate pedicle screw.

Statistical analysis. The success rate of the screw placement and the incidence of bone wall, blood vessel, nerve root and spinal cord injury were determined. The results were compared between the two groups using the chi-square test. $\mathrm{P}<0.05$ was considered to indicate a statistically significant difference.

\section{Results}

Clinical outcomes. After the removal of the pedicle screws, the channel wall of each pedicle screw was probed with a pedicle probe to check the integrity of the screw channel wall. The vertebral body was dissociated. The screw channel was directly observed after creation of the incision (Fig. 4).

In the guide device group, 2 of the 50 pedicles $(4 \%)$ were perforated (C3 vertebral artery injury and C5 nerve root injury, respectively). In the Abumi technique group, screw placement failed in 8 of the 50 pedicles $(16 \%$; $\mathrm{C} 3$ nerve root injury, $\mathrm{C} 3$ spinal cord injury, $\mathrm{C} 4$ nerve root injury, $\mathrm{C} 4$ vertebral artery injury, C5 nerve root injury, C5 vertebral artery injury, C5 spinal cord injury and C6 nerve root injury, respectively; Table I).

Statistical analysis. The chi-squared test revealed that the screws had been correctly implanted. However, the success

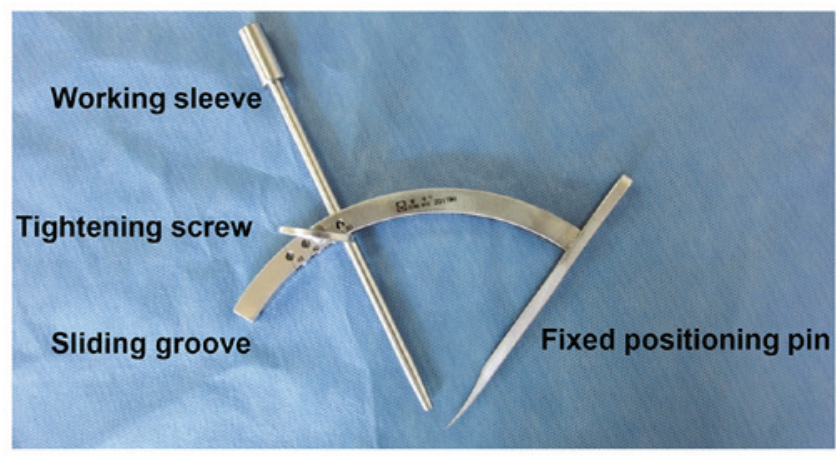

Figure 1. Subaxial cervical pedicle screw placement guide device. Fixed positioning pin, sliding groove, tightening screw and working sleeve are indicated.

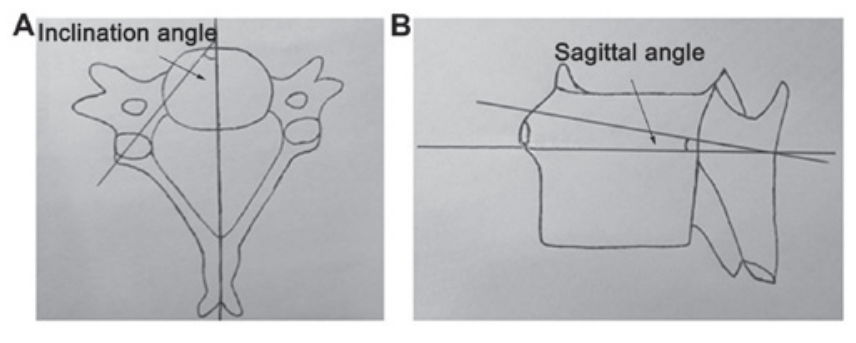

Figure 2. Inclination and sagittal angles of the pedicle detected by X-ray. (A) Inclination angle and (B) sagittal angle of the cervical pedicle.

rate of screw placement in the left pedicle (treated with the guide device) was significantly higher than that in the right pedicle (treated without the guide device; $\mathrm{chi}^{2}=4.00, \mathrm{P}<0.05$ ).

The incidence of perforation of the pedicle wall, as well as the vascular injury, nerve root injury and spinal cord injury resulting from it, was recorded in Table I.

\section{Discussion}

Prior to the development of subaxial cervical pedicle screw fixation, a large number of studies had assessed the three-dimensional morphology of the subaxial cervical pedicle. For instance, Reinhold et al (7) indicated that the coronal plane of the subaxial cervical pedicle is elliptical, and its inner width is shorter than its height. They also reported that the internal width of the pedicle gradually increased from $\mathrm{C} 3$ to $\mathrm{C} 7$. The width of $\mathrm{C} 3, \mathrm{C} 4, \mathrm{C} 5$ and $\mathrm{C} 6$ ranged from 4.2 to $5.6,4.4$ to $5.4,4.7$ to 5.6 and 4.7 to $6.5 \mathrm{~mm}$, respectively. However, the height of C3, C4, C5, C6 and C7 did not exhibit any substantial variation. Karaikovic et al (8) identified that only a small proportion of the subaxial cervical pedicles had no medullary cavity.

The front part of the subaxial cervical pedicle connects to the upper posterolateral vertebral body, and the back connects to the upper lateral mass. The entry site of the pedicle screw is located at the intersection of the vertical center line and horizontal midline through the upper $1 / 4$ of the lateral mass (9). The axis of the subaxial cervical pedicle at the C3-C4 level is relatively close to the upper endplate, but is slightly lower at C5-C7 (10). The anterior border of the upper endplate of the subaxial cervical pedicle is a slope. If the pedicle screw is 
A

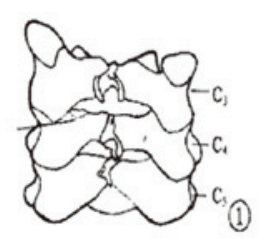

B

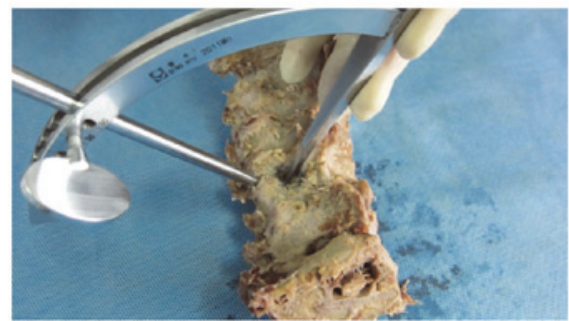

\section{C}

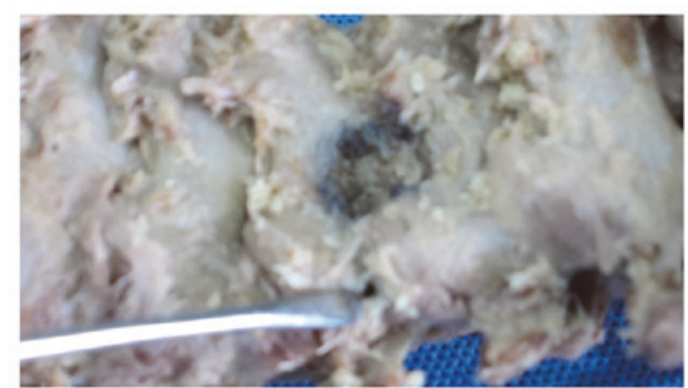

Figure 3. (A) Combined application of subaxial cervical pedicle screw placement guide device and 'keyhole' partial laminectomy and tapping technique. 1, Keyhole fenestration; 2, the nerve probe is used to probe the medial, superior and inferior borders of the pedicle; 3 , the line A-O indicates the direction of the working sleeve, line B-O indicates the direction of the fixed positioning pin, and point $\mathrm{O}$ indicates the intersection of the extension lines of the directions of the abovementioned objects. Point $b$ indicates the center of the medial wall of the pedicle and is used to fix the position of the end of the fixed positioning pin. The fixed positioning pin is utilized to probe the medial, superior and inferior borders of the pedicle and thus to identify the center of the pedicle. The direction of the pedicle is simultaneously probed and the direction of the working sleeve is adjusted accordingly. Finally, the pedicle screw is implanted. (B) 'Keyhole' fenestration is performed with the fixed positioning pin, which is used to probe the pedicle. (C) Keyhole fenestration and the mouth of the screw channel.
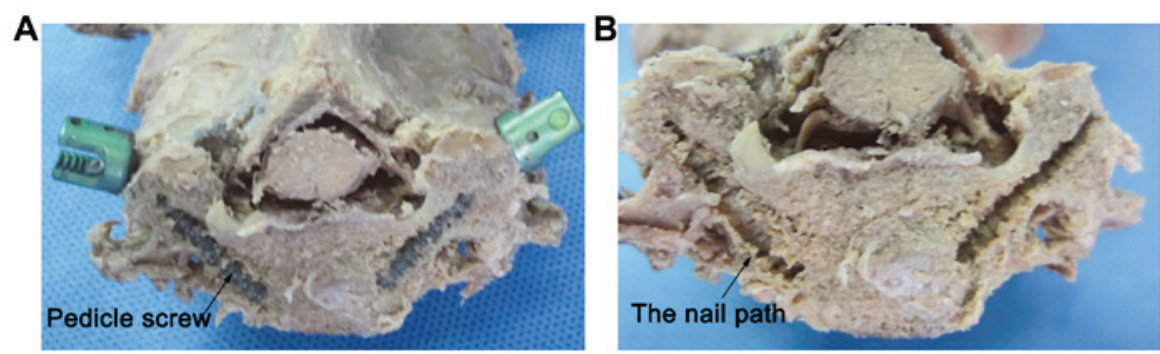

Figure 4. Screw channel following the creation of the incision. (A) Pedicle screw placement in cervical specimens. (B) The nail path after removing the screws.

too long, it may perforate the upper endplate when implanted along the axis of the pedicle.

In a study by Yukawa et al (11), the inclination angle of the subaxial cervical pedicle was 41.6-49.4 ${ }^{\circ}$. The effective width of the screw channel is maximal when the inclination angle of the subaxial cervical pedicle screw channel is identical to the inclination angle of the pedicle itself (12).

The medial wall of the subaxial cervical pedicle is thin $(13,14)$; thus, its resistance to external force is relatively low (15). Previous studies have suggested that the medial wall of certain subaxial cervical pedicles has a nourishing vessel entrance that results in congenital bone defects and reduced bone strength of the pedicle (16).

The height and width of the transverse foramen gradually increases from $\mathrm{C} 3$ to $\mathrm{C} 6$. The vertebral artery generally originates from the subclavian artery and enters the transverse foramen of $\mathrm{C} 6$, while some enter the transverse foramen at C3-C5 and C7 (17). The ertebral artery is surrounded by a venous sinus and distributes into the transverse foramen. The portion of the transverse foramen that is not traversed by the vertebral artery is relatively small (18).
The cervical nerve root tightly attaches to the inferior border of the intervertebral foramina and distributes forward and outward. The subaxial cervical pedicle, upper nerve root, and dural sac mostly attaches to one another at $1.0-2.5 \mathrm{~mm}$ from the lower nerve root. The cervical cord is packed within the dural sac and surrounded by cerebrospinal fluid, resulting in a great distance between the cervical cord and the pedicle (19). The anatomy of the subaxial cervical pedicle is controversial among researchers worldwide due to differences in ethnicity, sex and age, the dryness and storage period of the specimens and the methods of measurement $(20,21)$.

In 1991, Panjabi et al (22) determined the three-dimensional anatomical morphology of the human cervical pedicle and reported that pedicle screw fixation was successfully performed. In 1998, Wang et al (13) provided the measurement results of cervical specimens of 54 adult Chinese patients. The inclination angle at C3, C4, C5, C6 and C7 was 42.97, 44.28, $44.80,42.26$ and $35.23^{\circ}$, respectively. The sagittal angle (positive above the horizontal line and negative below the horizontal line) at C3, C4, C5, C6 and C7 was -5.16, -0.32, 2.40, 5.00 and $5.47^{\circ}$, respectively. In 1994 , Abumi (1) reported the application 
Table I. Incidence of failure in the two groups $(n=50)$.

\begin{tabular}{lcccr}
\hline Type & Control group & Guide device group & $X^{2}$ & P-value \\
\hline Perforation of pedicle wall $^{2}$ & $8(16)$ & $2(4)$ & 4.00 & 0.046 \\
Vertebral artery injury $^{\mathrm{a}}$ & $2(4)$ & $1(2)$ & & \\
Nerve root injury $^{\mathrm{a}}$ & $4(8)$ & $1(2)$ & & \\
Spinal cord injury $^{\mathrm{a}}$ & $2(4)$ & $0(0)$ & & \\
\hline
\end{tabular}

${ }^{\mathrm{a}}$ Consequence of perforation of the pedicle wall (subgroups).

of pedicle screw fixation in the treatment of subaxial cervical spine injury and obtained satisfactory clinical outcomes. In 2000, Abumi (23) reported on various complications associated with cervical pedicle fixation.

Ludwig et al (24) studied 67 pedicles at C3-C7 from 7 patients using the Abumi technique and obtained an overall pedicle cortical penetration rate of $40.3 \%$ (mild, $28.4 \%$; severe, $11.9 \%$ ) and an overall success rate of $88.0 \%$ (placement within pedicle, $59.7 \%$; slight penetration of cortex, 28.4\%). Abumi et al (23) performed cervical pedicle screw placement in 180 patients using a total of 669 screws. Post-operative CT revealed that 45 screws $(6.7 \%)$ penetrated the pedicle; 1 patient developed vertebral artery injury and 2 developed neurological symptoms.

The standards of subaxial cervical pedicle screw placement are not uniform. Five methods are currently utilized: The Abumi technique, the standard pedicle screw method, the partial laminectomy pedicle probe method, the dredging pipe method and three-dimensional computer navigation. As the classic Abumi technology has been used in our department for cervical screw placement, a group treated with the Abumi technique was used in the present study as a control. The Abumi technique involves partial removal of cortical and cancellous bone from the back of the lateral mass to expose the pedicle entrance, and the screw is then inserted either by direct visualization or under X-ray fluoroscopy.

The standard pedicle screw method involves identification of the pin entrance site and direction of entry according to anatomical landmarks. This technique may utilize a variety of positioning methods, all of which have common features. For instance, the pin entrance site is located within the outer quadrant superior to the cervical lateral mass. The inclination angle is $30-45^{\circ}$. The sagittal angle is negative at $\mathrm{C} 3-\mathrm{C} 4,0^{\circ}$ at C5 and positive at C6-C7 (25).

The dredging pipe method was described in 2001 by Karaikovic et al (8). Specifically, the cortical bone of the lateral mass is removed at the site of pin entrance by a rongeur or bone drill. The cancellous bone is removed by a curette with a 2- to 3-mm diameter rotated along the axis of the pedicle, exposing the flared pedicle entrance. Cancellous bone in the pedicle tube is then scraped in a rotating manner with the curette. The depth of the pedicle tube is $3-5 \mathrm{~mm}$ under direct visualization. If any resistance is encountered, the direction may be slightly adjusted. If screwing is still not possible, a new pin entrance site should be selected. Upon penetration of the pedicle tube, the screw is inserted along the medullary cavity. Ebrahein et al (26) first reported fenestration and laminectomy in 1997 . The vertebral plate was partially removed to
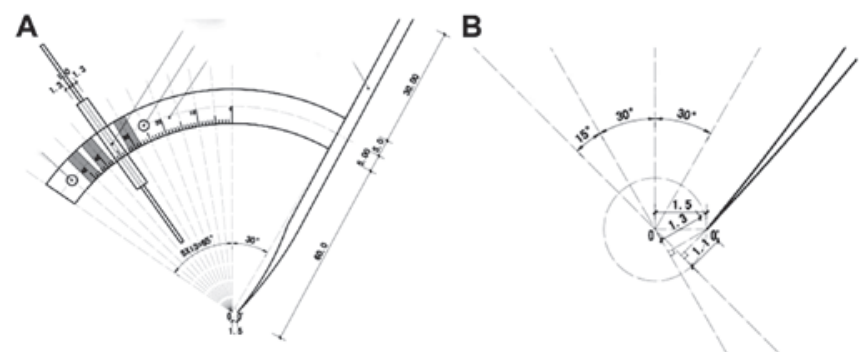

Figure 5. Distance ( $\left.\mathrm{OO}^{\prime}\right)$ between the center of the sliding groove and the tip of the fixed positioning pin. (A) Diagram of the cervical vertebra positioner. (B) Diagram of the angles guided by the cervical vertebra positioner (the coronal plane).

directly probe the position of or to expose the pedicle. Thus, the pedicle may can be safely inserted under direct visualization. Fenestration and laminectomy increases the accuracy of pedicle screw placement, but exposes the cervical cord, which may then be inadvertently injured. Three-dimensional computer navigation includes computer processing of the results of three-dimensional C-arm X-ray fluoroscopy and establishment of three-dimensional dynamic images to navigate the pedicle screw placement during the operation, thus increasing the safety of pedicle screw placement.

The criteria to evaluate the screw position differ among previous studies. Neo et al (15) classified the screw position into four grades: Grade 0, correct entrance of the screw channel along the axis of the subaxial cervical pedicle; grade 1, screw channel displacement of $<2 \mathrm{~mm}$; grade 2, screw channel displacement of $>2$ and $<4 \mathrm{~mm}$; and grade 3, screw channel displacement of $>4 \mathrm{~mm}$. Yoshimoto et al (27) defined partial screw perforation as a displacement distance of $<0.5$ times the screw diameter and complete screw perforation as a displacement a distance of $>0.5$ times the screw diameter. Kast et al (28) defined correct screw placement as $\leq 1 \mathrm{~mm}$ penetration of the subaxial cervical pedicle into the cortex, mild screw perforation as perforation that occurred lateral or anterior to the vertebral body or occupied the spinal lateral recess, did not contact the dural sac or extended outward to induce vertebral artery stenosis, and was $<1 / 4$ the diameter, and severe perforation as $>25 \%$ involvement of the vertebral artery or the occurrence of downward perforation-induced potential nerve compression or injury. Reinhold et al (7) classified screw placement results into four grades: Grade I, the subaxial cervical pedicle screw is located at the center of the pedicle; grade IIa, the subaxial cervical pedicle screw penetrates the 
medial wall of the pedicle, but the displacement is $<1 / 4$ of the screw diameter; grade $\mathrm{IIb}$, the displacement distance of the subaxial cervical pedicle screw is $>1 / 4$ of the screw diameter and the screw does not contact peripheral nerves or blood vessels; and grade III, the displacement distance is $>1 / 4$ of the screw diameter and the screw is attached to peripheral nerves and blood vessels.

The subaxial cervical pedicle screw placement guide device is able to probe the midpoint of the medial wall of the cervical pedicle using the fixed positioning pin. The guide sleeve was adjusted and fixed according to the angle measured by pre-operative X-ray fluoroscopy and multi-slice spiral CT. The working sleeve was connected to the sliding groove using the tightening screw. The ability to individually regulate the pin entrance angle for each vertebral body and guide the placement of the fixed positioning pin greatly assists clinicians in overcoming factors of uncertainty during screw placement. Thus, accurate and relatively safe screw placement may be achieved using the subaxial cervical pedicle screw placement guide device.

This guide device combined with the keyhole partial laminectomy and tapping technique allows the medial wall of the pedicle to be probed under direct visualization and facilitates assessment of the pin entrance site and direction. The design core concentrates on an arcuate sliding groove. The distance $\left(\mathrm{OO}^{\prime}\right)$ between the center of the sliding groove and the tip of the fixed positioning pin is $1.5 \mathrm{~mm}$ (Fig. 5). At various pin entrance angles, the vertical distance between the tip of the fixed positioning pin and the line of the pin entrance direction is $\geq 1.1 \mathrm{~mm}$ (it is precisely $1.1 \mathrm{~mm}$ when the pin entrance angle is $45^{\circ}$ ). After measuring the thickness of the cortical bone in the medial wall of the subaxial cervical pedicle, $>1.1 \mathrm{~mm}$ was the safest measurement that ensured the screw did not penetrate the medial wall due to the junction of the different intensities of cortical and cancellous bones in the pedicle. Only 2 cases of screw perforation occurred in the guide device group of the present study.

However, the design of the subaxial cervical pedicle screw placement guide device has certain limitations. The direction of the guide sleeve should be adjusted according to the angle measured by pre-operative X-ray fluoroscopy and CT. The inclination angle may be fixed by adjusting the position of the guide sleeve on the sliding groove, but the sagittal angle must be artificially adjusted according to the pre-operative evaluation. The outcomes are associated with the experience of the surgeon and the accuracy of the pre-operative CT evaluation. In addition, the precision of the guide device affects the direction of the guide sleeve. The midpoint of the medial wall of the pedicle is more readily identified by surgeons with greater experience. Based on the current study's operational experience in surgery, displacement may interfere with the direction of the guide device.

In the present study, two pedicles (4\%) in the guide device group were perforated, causing external injury in one case and inferior injury in the other. When the vertebral body was horizontally cut after completion of the procedure, it was revealed that the abovementioned injuries had been induced due to the direction from which the pin had been inserted, i.e., the pin entrance angle was too large. However, further in-depth studies are still required to address these issues.

In conclusion, application of the subaxial cervical pedicle screw placement guide device is more accurate and safer than previous techniques. This guide device overcomes certain anthropic factors during screw placement and ensures for an individually optimal pin entrance. However, further in-depth studies are required to reduce screw placement errors.

\section{Acknowledgements}

Not applicable.

\section{Funding}

No funding received.

\section{Availability of data and materials}

The analyzed data sets generated during the study are available from the corresponding author on reasonable request.

\section{Authors' contributions}

The final version of the manuscript has been read and approved by all authors, and each author believes that the manuscript represents honest work.

\section{Ethical approval and consent to participate}

The current study was approved by the Medical Ethics Board of Nanjing Hospital Affiliated to Nanjing Medical University (Nanjing, China). Informed consent was obtained from the families of the patients.

\section{Patient consent for publication}

Not applicable.

\section{Competing interests}

The authors declare that they have no competing interests.

\section{References}

1. Abumi K, Itoh H, Taneichi $\mathrm{H}$ and Kaneda K: Transpedicular screw fixation for traumatic lesions of the middle and lower cervical spine: Description of the techniques and preliminary report. J Spinal Disord 7: 19-28, 1994.

2. Nishizawa K, Mori K, Nakamura A and Imai S: Novel landmark for cervical pedicle screw insertion point from computed tomography-based study. Asian Spine J 11: 82-87, 2017.

3. Deng T, Jiang M, Lei Q, Cai L and Chen L: The accuracy and the safety of individualized 3D printing screws insertion templates for cervical screw insertion. Comput Assist Surg (Abingdon) 21: 143-149, 2016.

4. Uehara M, Takahashi J, Ikegami S, Kuraishi S, Futatsugi T and Kato H: Screw perforation rates in 359 consecutive patients receiving computer-guided pedicle screw insertion along the cervical to lumbar spine. Eur Spine J 26: 2858-2864, 2017.

5. Schnake KJ, Tropiano P, Berjano P and Lamartina C: Cervical spine surgical approaches and techniques. Eur Spine J 25 (Suppl 4): S486-S487, 2016.

6. Wu Z, Wei Y and Yu L: Analysis and prevention of the direction error of transpedicular fixation for the cervical spinal fracture. Chin J Spine Spinal Cord 14: 178-179, 2004 (In Chinese).

7. Reinhold M, Magerl F, Rieger M and Blauth M: Cervical pedicle screw placement: Feasibility and accuracy of two new insertion techniques based on morphometric data. Eur Spine J 16: 47-56, 2007. 
8. Karaikovic EE, Yingsakmongkol W and Gaines RW Jr: Accuracy of cervical pedicle screw placement using the funnel technique. Spine (Phila Pa 1976) 26: 2456-2462, 2001.

9. Bozbuga M, Ozturk A, Ari Z, Sahinoglu K, Bayraktar B and Cecen A: Morphometric evaluation of subaxial cervical vertebrae for surgical application of transpedicular screw fixation. Spine (Phila Pa 1976) 29: 1876-1880, 2004.

10. Chazono M, Soshi S, Inoue T, Kida Y and Ushiku C: Anatomical considerations for cervical pedicle screw insertion: The use of multiplanar computerized tomography reconstruction measurements. J Neurosurg Spine 4: 472-477, 2006.

11. Yukawa Y, Kato F, Yoshihara H, Yanase M and Ito K: Cervical pedicle screw fixation in 100 cases of unstable cervical injuries: Pedicle axis views obtained using fluoroscopy. J Neurosurg Spine 5: 488-493, 2006.

12. Richter M, Cakir B and Schmidt R: Cervical pedicle screws: Conventional versus computer-assisted placement of cannulated screws. Spine (Phila Pa 1976) 30: 2280-2287, 2005.

13. Wang D, Tang T and Huang S: Anatomical study and clinical application of pedicle screw fixation in lower cervical spine. Chin J Orthop 11: 659-662, 1998 (In Chinese).

14. Cervical pedicle screw instrumentation is more reliable with O-arm-based 3D navigation: analysis of cervical pedicle screw placement accuracy with O. European Spine Journal 27 2729-2736, 2018

15. Neo M, Fujibayashi S, Miyata M, Takemoto M and Nakamura T: Vertebral artery injury during cervical spine surgery: A survey of more than 5600 operations. Spine (Phila Pa 1976) 33: 779-785, 2008

16. Tse MS, Chan CH, Wong KK and Wong WC: Quantitative anatomy of C7 vertebra in Southern Chinese for insertion of lateral mass screws and pedicle screws. Asian Spine J 10: 705-710, 2016

17. Sanelli PC, Tong S, Gonzalez RG and Eskey CJ: Normal variation of vertebral artery on CT angiography and its implications for diagnosis of acquired pathology. J Comput Assist Tomogr 26: 462-470, 2002

18. Bruneau M, Cornelius JF, Marneffe V, Triffaux M and George B Anatomical variations of the V2 segment of the vertebral artery. Neurosurgery 59 (1 Suppl 1): ONS20-ONS24, 2006.

19. Simsek S, Uz A, Er U and Apaydin N: Quantitative evaluation of the anatomical parameters for subaxial cervical spondylectomy: An anatomical study. J Neurosurg Spine 18: 568-574, 2013.
20. Munusamy T, Thien A, Anthony MG, Bakthavachalam R and Dinesh SK: Computed tomographic morphometric analysis of cervical pedicles in a multi-ethnic Asian population and relevance to subaxial cervical pedicle screw fixation. Eur Spine J 24: 120-126, 2015.

21. Al-Saeed O, Marwan Y, Kombar OR, Samir A and Sheikh M: The feasibility of transpedicular screw fixation of the subaxial cervical spine in the Arab population: A computed tomography-based morphometric study. J Orthop Traumatol 17: 231-238, 2016.

22. Panjabi MM, Duranceau J, Goel V, Oxland T and Takata K: Cervical human vertebrae. Quantitative three-dimensional anatomy of the middle and lower regions. Spine (Phila Pa 1976) 16: 861-869, 1991

23. Abumi K, Shono Y, Ito M, Taneichi H, Kotani Y and Kaneda K: Complications of pedicle screw fixation in reconstructive surgery of the cervical spine. Spine (Phila Pa 1976) 25: 962-969, 2000.

24. Ludwig SC, Kowalski JM, Edwards CC II and Heller JG: Cervical pedicle screws: Comparative accuracy of two insertion techniques. Spine (Phila Pa 1976) 25: 2675-2681, 2000.

25. Aoude AA, Fortin M, Figueiredo R, Jarzem P, Ouellet J and Weber MH: Methods to determine pedicle screw placement accuracy in spine surgery: A systematic review. Eur Spine J 24: 990-1004, 2015.

26. Ebraheim NA, Xu R, Knight T and Yeasting RA: Morphometric evaluation of lower cervical pedicle and its projection. Spine (Phila Pa 1976) 22: 1-6, 1997.

27. Yoshimoto H, Sato S, Hyakumachi T, Yanagibashi Y and Masuda T: Spinal reconstruction using a cervical pedicle screw system. Clin Orthop Relat Res: 111-119, 2005.

28. Kast E, Mohr K, Richter HP and Börm W: Complications of transpedicular screw fixation in the cervical spine. Eur Spine J 15: 327-334, 2006.

This work is licensed under a Creative Commons Attribution-NonCommercial-NoDerivatives 4.0 International (CC BY-NC-ND 4.0) License. 